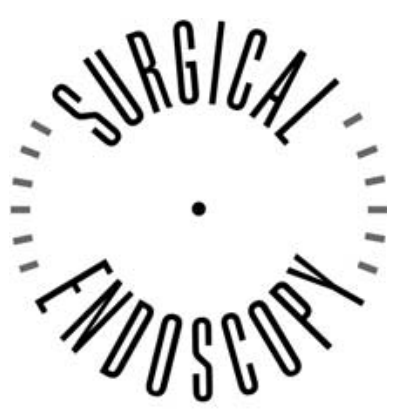

and Other Interventional Techniques

\title{
Ergonomic assessment of the static stress confronted by surgeons during laparoscopic cholecystectomy
}

\author{
A. Vereczkei, ${ }^{1}$ H. Feussner, ${ }^{2}$ T. Negele, ${ }^{2}$ F. Fritzsche, ${ }^{3}$ T. Seitz,${ }^{3}$ H. Bubb,${ }^{3}$ Ö. P. Horváth ${ }^{1}$ \\ ${ }^{1}$ Department of Surgery, Faculty of Medicine, University of Pécs, Ifjuság utja 13, 7624 Pécs, Hungary \\ 2 Department of Surgery, Arbeitsgruppe MITI, Technische Universität München, Klinikum rechts der Isar, Ismaningerstr. 22, D-81675 Munich, \\ Germany \\ ${ }^{3}$ Department of Ergonomics, Technische Universität München, Boltzmannstr. 15, D-85747 Garching, Germany
}

Received: 25 June 2003/Accepted: 04 January 2004/Online publication: 12 May 2004

\begin{abstract}
Background: Only a few reports on static strain in the spine, neck, and head of the surgeon are available, describing it as "distinctly harmful." The aim of this study was to objectively prove the static burden during laparoscopic operations. For this, new industrial software called PCMAN was used, capable of measuring and comparing the postures of the surgeon at different monitor placements.

Methods: Two simultaneous and synchronized video recordings of laparoscopic cholecystectomies (LC) were done using miniDV digital camcorders with the cameras standing at a $90^{\circ}$ angle to each other. Twenty operations were performed using two different placements of the monitor. In 10 cases, the monitor was placed at the patient's head in the center, and in 10 cases at the left side of the patient. Using the time codes of the recordings, different steps of the operation were identified, and the duration of these measured in seconds. Very characteristic, longer lasting postures were imported to and analyzed with the software. Results of the different setups were compared to each other, and to an "ideal" comfort posture.

Results: During the intermediate steps of the operations the rate of static phases is significantly higher. Measuring the typical postures of these phases the trunk and head are significantly more rotated and bent than in comfort positions. When the monitor was at the side of the patient facing the surgeon, results were closer to the comfort posture.

Conclusions: It was proven that surgeons are confronted by significant static burden during LC. The software used was able to evaluate objectively the static posture
\end{abstract}

of the surgeon during series of LC. Results also confirmed that the position of monitors significantly influences the surgeon's posture. Best setups for the whole team can be achieved by adjustable multiple monitor systems.

Key words: Ergonomics — Static stress - Posture analysis

The benefits that minimally invasive surgery brought to patients have been studied since its very first introduction. Reduction of pain, shorter recovery time and hospital stay, and earlier restitution of normal physiological markers have been confirmed as the most significant of these advantages. It also became clear that minimally invasive operations could also cause harm to patients if used uncritically [5]. The phenomenon, however, that laparoscopic surgery may also harm laparoscopic surgeons was only discovered later and is now being investigated worldwide $[1-4,6]$. These disadvantages of laparoscopic procedures are mainly caused by the nonergonomic design of surgical instruments and environment of the operating room. As laparoscopic surgery became more and more advanced and complex, so did the duration of procedures, and in proportion to this the mental and physical stress increase. In spite of this, up to now no significant changes have been made to the operating-room environment, which was originally designed for conventional operations $[3,9]$.

Ergonomics, a relatively new field of science, gained wide popularity in industrial engineering. It is commonly accepted that nonergonomic circumstances in a working environment lead to a drop in quality and productivity. Accordingly, a growing sum of money is being spent on ergonomic research, and the results are serious factors in the design of new products. Reflecting 


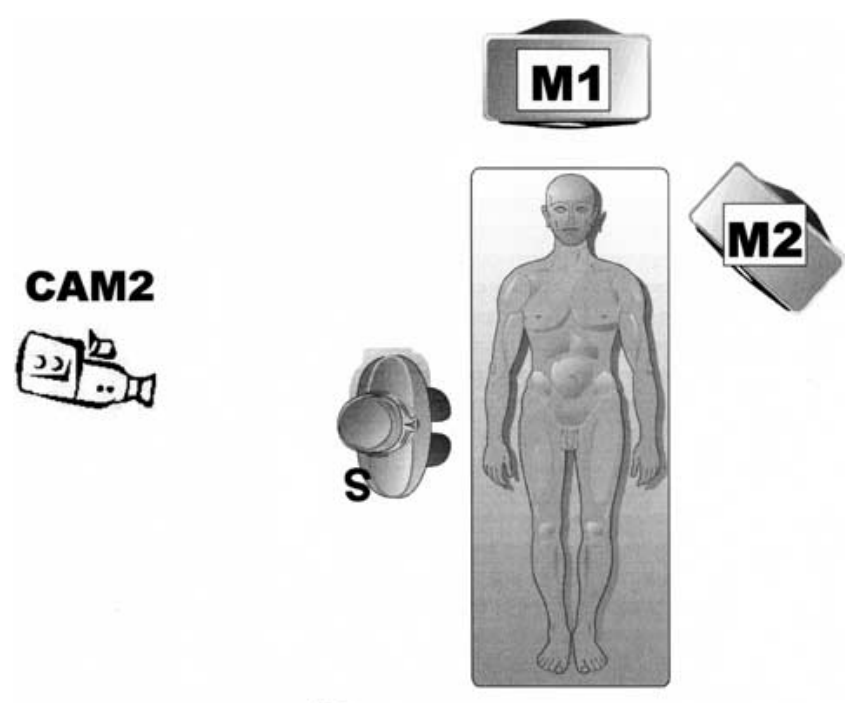

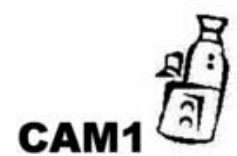

Fig. 1. Operative setup with cameras (CAM) and different monitor positions (M1 and M2).

these requirements, new industrial software tools have been developed to measure the posture and movement of the human body very accurately, without using any markers [11]. As only a few reports on static stress in the spine, neck, and head of the surgeon are available, the aim of this study was to objectively prove this burden during laparoscopic operations. For this new software (called PCMAN) was used, which was previously proven to be capable of posture tracking without markers under laboratory circumstances in the vehicle industry.

\section{Materials and methods}

From 1 November to 31 December 2002, 20 noncomplicated laparoscopic cholecystectomies (LC) were recorded at the Department of Surgery of the Technische Universität München, Klinikum rechts der Isar. Two experienced surgeons alternately performed all procedures. Two simultaneous and synchronized video recordings were done using miniDV digital camcorders, with wide-angle optics and night-shot function. The surgeon performing the operation was in the center of the recordings, with the cameras standing at a $90^{\circ}$ angle to each other (Fig. 1). The operations were performed using two different placements of the monitor. In 10 cases, the monitor was placed at the patient's head in the center (position 1), in the other 10 cases at the left side of the patient (position 2) (Fig. 1.). At the end of each procedure, a calibrating body standing at the previous position of the operating surgeon was recorded, without moving the cameras in the meantime (Fig. 2). So-called comfort postures were also recorded, when the surgeon was standing at the operating table in the most comfortable resting position (Fig. 3).

Later, using the time codes of the recordings, all were analyzed, different steps of the operation were identified, and the duration of these steps was measured in seconds. It turned out that during the operation the trunk, head, and neck (torso) remain unmoved most of the time, while upper and lower extremities are doing most of the actions. Accordingly, the static posture and dynamic periods of the surgeon's torso were also measured in seconds. Very characteristic, longer lasting postures were digitalized into graphic files. Always, pairs
CAM1

CAM2

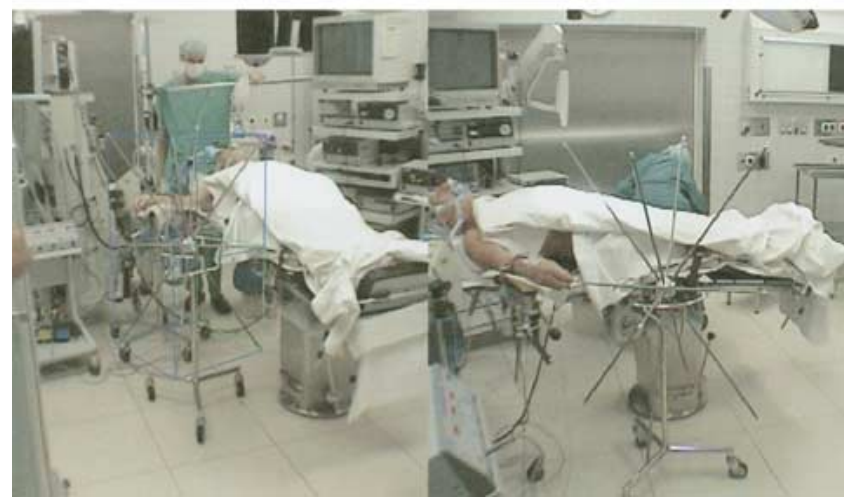

Fig. 2. Calibrating body at the operating table with PCMAN calibrating module.

\section{CAM1}

\section{CAM2}

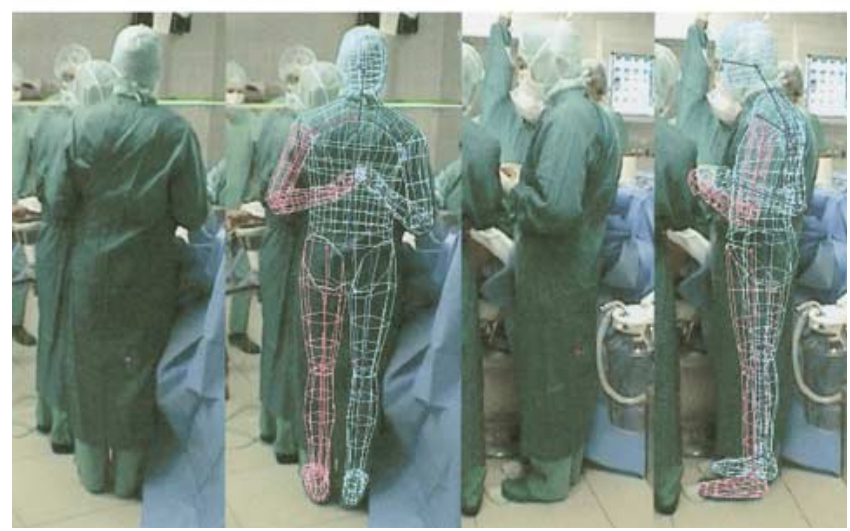

Fig. 3. Comfort positions, with PCMAN measurement modules.

of pictures, taken simultaneously from the two different viewpoints, were produced. Using the similar recordings of the calibrating body as a referral point, the digitalized pictures were analyzed with the software PCMAN, which was proved to accurately measure the rotation, lateral, and anteroposterior movements of joints and parts of the body. The pairs of pictures were imported, and 17 orientation points of the body - required by the program - were marked on both. After this a rough tracking of the posture was prepared automatically, which had to be refined step-by-step manually, until the closest similarity was achieved (Fig. 4). From the data collected by the software, those referring to the hip, spine, neck, and head were selected. In the program, five reference points determine the position of the hip and spine. The declinations at these points were added axis by axis to get the complete declination of the trunk (Fig. 5). This was also carried out integrating the neck and head movements. The pairs of pictures of the comfort postures were similarly analyzed. For statistical analysis the unpaired $t$-test of the program SPSS ver. 11.0. was used; the significance level was set at 0.05 .

\section{Results}

Reviewing the videotapes, nine different steps of LC were identified (Table 1). We found that these can also be described as steps consisting of mainly static or dynamic phases regarding the torso. The dynamic phases were at the beginning and at the end of the operations: insufflation, trocar placement, gallbladder extraction, drainage and wound closure. On the other hand, the 
CAM1 CAM2

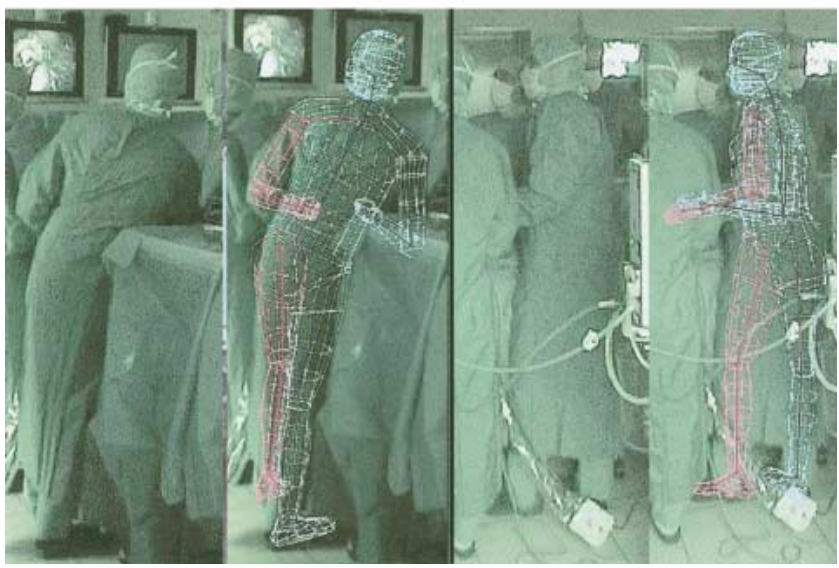

Fig. 4. Intraoperative images, with PCMAN measurement modules.

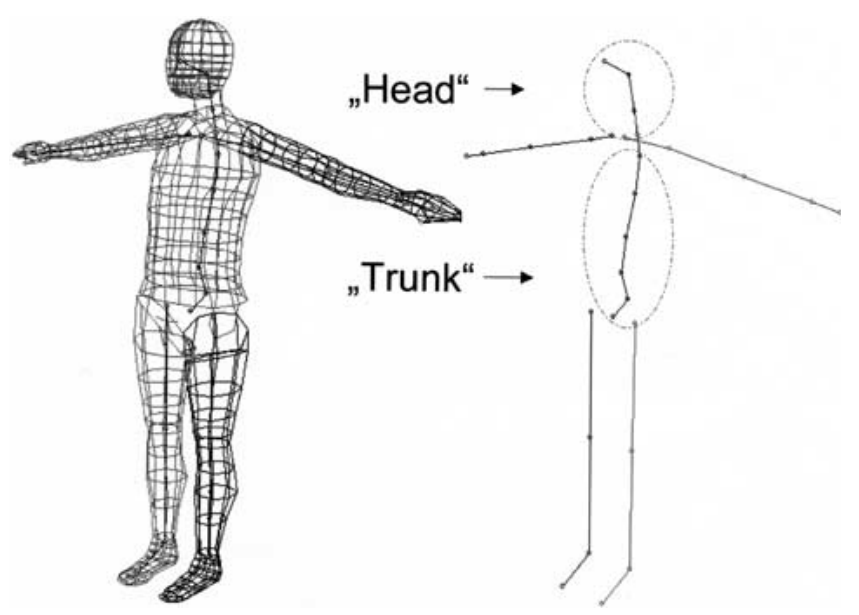

Fig. 5. Map of body elements; the regions of interests "trunk" and "head" are marked.

Table 1. Steps of laparoscopic cholecystectomy, with mean durations and mean rates of static phases

\begin{tabular}{|c|c|c|c|c|c|c|c|c|c|}
\hline & Insufflation & $\begin{array}{l}\text { Trocar } \\
\text { placement }\end{array}$ & Preparation & Clipping & $\begin{array}{l}\text { Gallbladder } \\
\text { dissection }\end{array}$ & $\begin{array}{l}\text { Coagulation } \\
\text { irrigation }\end{array}$ & $\begin{array}{l}\text { Gallbladder } \\
\text { extraction }\end{array}$ & $\begin{array}{l}\text { Irrigation } \\
\text { drainage }\end{array}$ & $\begin{array}{l}\text { Wound } \\
\text { closure }\end{array}$ \\
\hline $\begin{array}{l}\text { Mean } \\
\text { duration } \\
(\mathrm{sec})\end{array}$ & 242 & 166 & 505 & 299 & 362 & 201 & 349 & 268 & 376 \\
\hline $\begin{array}{l}\text { Duration of } \\
\text { static } \\
\text { phases }(\%)\end{array}$ & 5.4 & 11.6 & 91 & 63.4 & 78.4 & 68.2 & 19.3 & 41 & 23.5 \\
\hline
\end{tabular}

Table 2. Positions of trunk and head at different monitor placements (values in degrees)

\begin{tabular}{|c|c|c|c|c|c|c|}
\hline & \multicolumn{3}{|c|}{ Trunk } & \multicolumn{3}{|c|}{ Head } \\
\hline & Rotation +++ & Lateral bending +++ & Posterior bending & Rotation +++ & Lateral bending & Posterior bending \\
\hline Comfort & $8.95 \pm 5.13$ & $11.33 \pm 8.69$ & $1.82 \pm 2.62$ & $1.7 \pm 5.33$ & $1.4 \pm 3.63$ & $2.87 \pm 1.79$ \\
\hline Position 1 (M1) & $53.05 \pm 8.51^{* * *}$ & $35.53 \pm 4.88^{* * *}$ & $3.77 \pm 4.08$ & $36.71 \pm 12.07^{* * *}$ & $-23.81 \pm 9.05^{* * *}$ & $7.97 \pm 5.29$ \\
\hline Position 2 (M2) & $32.21 \pm 6.73^{* * *}$ & $22.93 \pm 7.81^{*}$ & $2.4 \pm 5.19$ & $-11.41 \pm 4.86^{* *}$ & $-19.45 \pm 6.81^{* * *}$ & $3.73 \pm 3.54$ \\
\hline
\end{tabular}

${ }^{* * *}, p<0.001 ;^{* *}, p<0.01 ;^{*}, p<0.05, \mathrm{M} 1$ and M2 compared to comfort;,$+++ p<0.001$, M1 and M2 compared to each other

phases of preparation, clipping, gallbladder dissection, and coagulation-suction consisted of long static-posture episodes, disrupted only by short intervals of instrument changes, when not only the extremities but also the torso moved.

The duration of the operations was $2768 \mathrm{sec}(1670$ 3790) on average. Considering the whole procedure, the rate of static periods was $44.6 \%(59.5-28.7 \%)$ and the rate of dynamic periods $55.4 \%(71.3-40.5 \%)$ on average. If we only look at the middle steps consisting mainly of static phases these values are $75.25 \%(86.8-72.5 \%)$ for the static, and $24.75 \%(27.5-13.2 \%)$ for the dynamic phases, with a statistically significant difference $(p<$ $0.01)$. As the beginning and closing steps of all laparoscopic operations are the same (insufflation, trocar placement, tissue extraction, and wound closure), the difference in the static/dynamic phase rate of different procedures is determined by the period lying between these steps, which are mainly static. With more and more complex operations lasting for hours, this period gets longer and longer, thus increasing the rate of static phases for the whole procedure. To deal with the identified unphysiologic postures [1,9], only these static phases were considered on further analysis. It became clear that the posture of the torso is rather constant and is independent of the actual action of the upper extremities (preparation, clipping, gallbladder dissection, or coagulation-suction). The explanation for this is the fixed position of trocars. To access these, a certain torso posture in required. Later on only the upper (instruments) or lower extremities (foot-pedal) have to be moved to perform the operation; the torso remains fixed. However, this fixed position has to be changed in order to take other instruments, but these dynamic intervals last no longer than $40 \mathrm{sec}(10-20 \mathrm{sec}$ mostly). This fixed position was apparently very unphysiologic (Fig. 4). The issue of the next investigation was whether this posture deviation could be measured objectively with the program. The data of the postures of the two surgeons with the same setup were first compared. As no 
significant differences were found, all data for the same monitor settings were merged and compared to the comfort postures, then to each other (Table 2). Compared to the comfort position the trunk and the head was significantly more rotated and bent to the side $(p<$ 0.001 ), when the monitor was placed in the center (position 1). Analyzing the other placement of the monitor (position 2), less significant differences were found; however, the trunk was also more rotated $(p<0.001)$ and bent to the side $(p<0.05)$, which was also true for rotation $(p<0.01)$ and bending $(p<0.001)$ of the head. Comparing the different monitor positions to each other, the trunk and the head were more rotated $(p<$ $0.001)$, and the trunk bent to the side $(p<0.001)$ at position 1. These results confirm the findings of the visual observations. When the surgeon is standing at the side of the patient, the trunk has to be rotated and bent to access the trocars with both hands (Fig. 4). The lateral bending of the trunk is compensated with the bending of the neck to the opposite direction. To see the monitor the head has to be rotated to its location.

\section{Discussion}

Nowadays it is obvious, that the setup of laparoscopic operations is mostly not ergonomic. This situation increases mental and physical stress equally. Physical stress can cause eye strain, neck, shoulder, and wrist pain or stiffness, and premature fatigue, thus degrading of performance] [2, 6, 9]. Even damage to the nerves of the thumb and thenar may occur, causing the so-called "laparoscopist's thumb" [8, 10]. Physical stress is built up of static and dynamic components. Recent studies concentrated mainly on the dynamic part, objectively confirming the unphysiologic movements and increased muscle activity resulting in premature fatigue of the upper extremities $[3,9,10]$. Static strain was mostly indirectly assessed, as by force-plate measurements, or by manual notes of the surgeon's posture, observed by outside investigators and described it as "distinctly harmful" [9]. These investigations were carried out mostly under experimental circumstances, or with only a few clinical cases.

There are only a few studies analyzing the surgeon's posture and the monitor position. In a recent one [12], only the posture of the neck and head was measured using printed frames and three-dimensional, markerguided infrared video recordings. This study proved that the position of the monitor significantly influences head and neck torsion and bending during laparoscopic cholecystectomy. With the PCMAN software not only the neck and head, but also the whole body can be measured, including the spine, which is also prone to significant static burden. It is also new, that this method does not require special markers, wires, or sensors attached to body. Such an outfit can be uncomfortable and may drift during movements, thus influencing the results [10]. We could prove that there is a significant difference between the surgeon's postures at comfort and operative positions. This operative posture in the analyzed procedure was characterized by the rotation and bending of the upper trunk and the head. This can be one of the causes of static stress, which is responsible for the shoulder, back, and neck stiffness or pain experienced during long interventions. The software was also able to measure differences when the monitor was placed in other positions. These data suggest that the operative posture is closer to the comfort one, when the surgeon, the trocars, and the area of manipulation are aligned in the same vector, and the monitor is placed facing the surgeon. This favorable placement of the monitor is also in accordance with task performance studies [7]. Theoretically the deviations necessary for the trocar access could be eliminated if the surgeon was standing between the legs of the patient. The deviations to see the picture of the laparoscope could also be minimized with freely adjustable monitor systems. Naturally the "ideal" localization of the surgeon cannot always be carried out. On the contrary, the best monitor positioning could be accomplished by using roof-mounted multiple monitor systems that are already available and allow the best setting for the surgeon and the assistant to be freely adjusted. This also could be a guideline for the design of operating rooms where laparoscopic surgery is also performed.

However there are still some problems regarding the software that must be refined. In the vehicle industry this software was used for both motion and posture analysis. Under operative circumstances it is only capable of posture analysis, because continuous and accurate image tracking is hindered by the obstruction of the view by the assistants or overlapping body parts, and clothing that only loosely fits the body. The lack of accurate automatic measurement of single postures is also due to the same reason. It requires a lot of manual settings, which is time-consuming, so it might be even user dependent. As no other software capable of simultaneous motion and posture measurements without markers for operative purposes is available, significant progress in posture and motion analysis during laparoscopic surgery requires the development of tracking tools.

\section{References}

1. Berguer R, Rab GT, Abu-Ghaida H, Alarcon A, Chung J (1997) A comparison of surgeons' posture during laparoscopic and open surgical procedures. Surg Endosc 2: 139-142

2. Berguer R, Forkey DL, Smith WD (1999) Ergonomic problems associated with laparoscopic surgery. Surg Endosc 5: 466468

3. Berguer R, Smith WD, Chung YH (2001) Performing laparoscopic surgery is significantly more stressful for the surgeon than open surgery. Surg Endosc 10: 1204-1207

4. Berquer R, Smith WD, Davis S (2002) An ergonomic study of the optimum operating table height for laparoscopic surgery. Surg Endosc 3: 416-421

5. Cuschieri A (1995) Whither minimal access surgery: tribulations and expectations. Am J Surg 1: 9-19

6. Emam TA, Frank TG, Hanna GB, Cuschieri A (2001) Influence of handle design on the surgeon's upper limb movements, muscle recruitment, and fatigue during endoscopic suturing. Surg Endosc 7: $667-672$ 
7. Hanna GB, Shimi SM, Cuschieri A (1998) Task performance in endoscopic surgery is influenced by location of the image display. Ann Surg 4: 481-484

8. Horgan LF, O'Rionda DC, Doctor N (1997) Neoropraxia following laparoscopic procedures: an occupational injury. Min Inv Ther Allied Technol 6: 33-35

9. Nguyen NT, Ho HS, Smith WD, Philipps C, Lewis C, De Vera RM, Berguer R (2001) An ergonomic evaluation of surgeons' axial skeletal and upper extremity movements during laparoscopic and open surgery. Am J Surg 6: 720-724
10. Person JG, Hodgson AJ, Nagy AG (2001) Automated high-frequency posture sampling for ergonomic assessment of laparoscopic surgery. Surg Endosc 9: 997-1003

11. Seitz T, Balzulat J, Bubb H (2000) Anthropometry and measurement of posture and motion. Int $\mathbf{J}$ Indust Ergonomics 25: 447-453

12. van Veelen MA, Jakimowicz JJ, Goossens RHM, Meijer DW, Bussmann JBJ (2002) Evaluation of the usability of two types of image display systems during laparoscopy. Surg Endosc 16: 674678 